\title{
Experience of an informational system design with an integrated pollution transport model on a water surface
}

\author{
Sergey Rychkov ${ }^{1}$, Vyacheslav Solbakov ${ }^{2}$, and Anatoly Shatrov ${ }^{1,3, *}$ \\ ${ }^{1}$ Vyatka State University, 610000, Moskovskaya str., 36, Kirov, Russian Federation \\ ${ }^{2}$ Federal Research Centre "Computer Science and Control" of Russian Academy of Sciences, \\ 119333 , 4, b. 2, Vavilova str., Moscow, Russian Federation \\ ${ }^{3}$ Kirov State Medical University, 610027, 112, Karl Marx str., Kirov, Russian Federation
}

\begin{abstract}
The use of informational systems to estimate the required amount of chemicals for the dispersion of surface pollution is a fruitful approach in implementations of emergency response plans. A mathematical model of the processes of destruction of pollution on a water surface is an integral part in such a system and a source of accumulation of information about the evolution of pollution in various environmental conditions and characteristics of the source of pollution. Examples of the results of modeling the processes of surface pollution propagation when exposed to surface waves are presented, as well as a set of stored information for estimating the required amount of reagents.
\end{abstract}

\section{Introduction}

The problems of transport of contaminants on the surface of water bodies are determined by environmental conditions. On the surface of the water, oil spills pose a great danger. Surface waves are an important factor in the destruction of oil spills. In this work, we consider mathematical models of surface wave formation and the evolution of oil spills. Assessing the use of response tools, in particular dispersants, choosing an appropriate response strategy, as well as assessing the required amount of reagents, is a particular task in the implementation of activities for removal the consequences of oil spills. A fairly detailed description of the current situation in the field of operational modelling of the evolution of oil spills at sea, as well as an analysis of environmental problems, is presented in the review [1]. This paper describes the process of creating an information system for modeling and monitoring the processes of oil spill elimination, and describes its main elements. The software suit consists of an expert system based on a database formed from the calculation results for making a decision to eliminate oil spill and a calculation module for forming the database. The database contains a description of scenarios of possible oil discharges, which correspond to the physicochemical properties of oil and the values of parameters of hydrometeorological environmental conditions, as well as the results of calculations of oil spill characteristics. The system also operates a module that implements

\footnotetext{
*Corresponding author: shatrov@vyatsu.ru
} 
an algorithm for calculating the required amount of dispersant for spill processing based on information stored in the database.

\section{Methods}

\subsection{Methods of modeling and monitoring for transport of pollutants}

Surface waves are the main and essential factor for dispersing oil spills. Currents in the sea are determined by atmospheric wind action, and by sea-level fluctuations in the form of waves. There are a large number of models describing surface waves in marine areas. These include spectral models that use the boundary layer equation with the Hasselman force and the turbulent energy transfer equation for closure to parameterize the effects of sea surface waves [2]. The basis of such models is the concept of the description of the wave oscillations proposed by Hasselmann [3]. Numerical models for calculating surface waves based on the Lagrangian approach for describing the free boundary use recently the turbulent energy transfer and turbulent dissipation equations for closing the system $[4,5,6$, 7]. In these models, special attention is paid to the interaction of wave and shear currents and their influence on the formation of turbulent energy at the atmosphere - sea surface separation boundary. There are many papers devoted to experimental verification of the models used. The paper [8] presents the results of field and laboratory studies of the mechanism of destruction of surfactant oil slicks by intense surface waves. The mechanism of oil slick destruction in the gravitational waves collapse zone is modeled in a controlled laboratory experiment with artificial surface slicks. Based on the results of field observations, it is shown that in the areas of collapsing wave crests, the slick is destroyed due to vertical mixing associated with the generation of turbulence during the collapse. The influence of surface waves on the formation of turbulence as one of the factors of inhomogeneity and destruction of waves is experimentally shown in works [9-11] Semiempirical models for evaluating the effect of natural dispersion on the vertical transport of pollutants into the water depth are widely used. The calculation of the vertical dispersion of oil in water, that is, the penetration of oil droplets into the surface layer of the sea due to the collapse of waves, resulting in the formation of an oil-in-water emulsion, can be performed, for example, using the method [12], [13].

$$
Q_{\text {disp }}=C_{\text {oil }} D^{0.57} f_{S} F_{w c} d_{0}^{0.7} \delta d
$$

where $Q_{\text {disp }}$ - oil stream $(\mathrm{kg} / \mathrm{m}) ; f_{S}$ - sea surface, covered by oil; $d_{0}$ - mean diameter of drops; $\delta d$ - interval of drop diameters; $C_{o i l}$ - a parameter, depending on oil properties; $D$ - dissipation of wave energy on square entity:

$$
D=0.0034 \rho_{w} g H_{r m s}^{2}, \quad H_{r m s}=\frac{H_{\text {wave }}}{\sqrt{2}}
$$

where $H_{\text {wave }}$ - height of wave; $\rho_{w}$ - water density; $F_{w c}$ - part of sea surface, covered by whitecaps in time entity:

$$
F_{w c}=0.032 \frac{\left(W-W_{c}\right)}{T_{\text {wave }}}
$$


where $\mathrm{W}$ - wind speed; $W_{s}$ - wind speed of whitecaps beginning $(5 \mathrm{~m} / \mathrm{s}) ; T_{\text {wave }}$ - wave period. It is believed that oil is driven into the water in the form of drops, but large drops immediately float to the surface. The threshold size of drops that remain in the water is estimated at $70-150 \mu m$ [12]. The parameter $C_{\text {oil }}$ depends on the properties of the oil and is determined experimentally. In the work [11], experiments were carried out with three types of oil, as a result, the value of parameter a was estimated from 510 to 1800 . It is noted that the parameter $C_{\text {oil }}$ strongly depends on the viscosity of the oil $[11,12]$. The parameterization suggested in the model MOHID is possible [13]:

$$
C_{\text {oil }}=-312.25 \ln \left(\frac{\mu_{\text {oil }}}{\rho_{\text {oil }}}\right)+2509.8
$$

where: $v=\left(\frac{\mu_{\text {oil }}}{\rho_{\text {oil }}}\right)-$ is kinematic viscosity of oil. If $C_{o i l} \leq 0$ it is supposed that $C_{o i l} \leq 0$ and dispersion is absent.

The main problem of such models in describing a surface wave remains the complexity of describing a free surface. Thus, one of the components of currents in the spill zone, namely surface oscillations, is described in insufficient detail by marine hydrodynamic models. This is especially important from the point of view of describing the process of dispersion of oil spills. One way to correct the situation may be to introduce additional artificial modeling of the distribution of oil components in the resulting emulsion. For this purpose, a wave model is constructed taking into account the dynamics of particles formed when oil is dispersed by solid sorbents. The resulting mixture is a dispersed system consisting of immiscible liquids. One of them, the polar liquid, is conventionally called the water phase, the other-the non-polar liquid, the oil phase. The continuous phase is called the dispersion medium, the phase that is in the form of individual droplets of small size is called the dispersed phase. The method of particle dynamics is used to describe the oscillations of a set of phases in the form of surface waves.

\subsection{Simulation model of surface waves}

The simulation is performed in a two-dimensional vertical-longitudinal space. The method of particle dynamics used is essentially a description of interacting particle-oscillators [14]. Two media (seawater and oil) are represented as separate spherical elements. The nature of the interaction between elements is considered viscoelastic. The properties of media (density, modulus of elasticity, internal friction) are embedded in the primary properties of the individual elements (mass, diameter, stiffness and viscosity coefficients, interaction restriction radii). The motion of each element in space is described by Newton's second law, which results in the following system of equations:

$$
\begin{aligned}
& m_{e} \frac{d^{2} x_{i}}{d t^{2}}=\sum_{j=1(j \neq i)}^{N_{e}}\left(\left(\left\{\begin{array}{l}
\left.\left.\mu\left(d_{e}-r_{i j}\right) \frac{x_{i}-x_{j}}{r_{i j}}, r_{i j}<d_{0}\right)+k\left(r_{i j}-d_{1}\right)\left(v_{x i}-v_{x j}\right)\right), \\
0, r_{i j} \geq d_{0}
\end{array}\right)\right.\right. \\
& m_{e} \frac{d^{2} y_{i}}{d t^{2}}=-m_{e}+\sum_{j=1(j \neq i)}^{N_{e}}\left(\left(\left\{\begin{array}{l}
\mu\left(d_{e}-r_{i j}\right) \frac{y_{i}-y_{j}}{r_{i j}}, r_{i j}<d_{0} \\
0, r_{i j} \geq d_{0}
\end{array}\right)+k\left(r_{i j}-d_{1}\right)\left(v_{y i}-v_{y j}\right)\right) .\right.
\end{aligned}
$$


Thus, the wave model is a collection of interacting particles-elements of two phases [15, 16]. The list of the variables and the parameters of the model as follows:

$m_{e}$ - mass of elements oil-water; $d_{e}$-diameter (size) of elements oil-water; $x_{i}, y_{i}$-coordinates of elements for wave model; $\mathrm{t}$ - the current time; $N_{e}$ - the number of elements in the model, $\mathrm{k}$ - the stiffness coefficient (of the elastic interaction) in the model; $\mu$ - the viscosity coefficient (of the dissipative interaction) in the model; $d_{0}$ - the dimension of the elastic interaction region; $d_{1}$ - the dimension of the dissipative interaction region; $v_{x i}, v_{y i}$ the velocity components of the i-th element; $r_{i j}$ the distance between the centers of the i-th and j-th elements.

To create waves, the left and right borders of the modeling space of the original rectangular shape (of size $L_{X} \times L_{Y}$ ) move according to the law:

$$
X_{L g}=A \frac{y}{h} \sin (2 \pi f t), \quad X_{R g}=L_{X}+X_{L g}
$$

$X_{L g}, X_{R g}$, define the motion of the left and right borders of the modeling space respectively; $f$ - the frequency of the wave oscillations; $A$ - amplitude of the horizontal oscillations of the water surface; $h$ - the depth of the modeling space.

\subsection{Mathematical model for evolution of oil spills}

Modern mathematical models of the evolution of the oil spill are traditionally described on the basis of the Navier-Stokes equations in the approximation of a thin layer of light liquid on the open surface of a heavier one [17]. The equations of momentum balance and continuity are presented in the form:

$$
\begin{gathered}
\frac{\partial}{\partial t} \bar{u}_{o}+\bar{u}_{o}\left(\operatorname{div} \bar{u}_{o}\right)=-g^{\prime} \nabla h-\left(\bar{\tau}_{w}+\bar{\tau}_{a}\right) /\left(\rho_{o} h\right)+\bar{f} \\
\frac{\partial}{\partial t} h+\operatorname{div} \bar{u}_{o} h=-Q_{\Sigma} / \rho_{o}
\end{gathered}
$$

where are: $h$ - oil slick thickness, $\bar{u}_{o}$ - oil velocity, $g$ - acceleration of gravity, $\rho_{0}$ - oil density, $\rho_{\mathrm{w}}$ - waterl density, $g^{\prime}=g\left(\rho_{w}-\rho_{o}\right) / \rho_{w}, \bar{\tau}_{w}, \bar{\tau}_{a}$ - friction stresses at the surface of oil-air and oil-water $\bar{f}$ - surface tension forces, $Q_{\Sigma}$ - resulting specific mass flow from the oil slick. During the evolution of spill on the surface of the sea, the viscosity, density, and other physicochemical properties of surface pollution are changing. There are two main physical processes leading to a decrease in the amount of oil on the surface of the sea: evaporation and dispersion [13, 18, 19], dissolution is also involved in this process, to a much lesser extent. A similar description of the parameterizations used is given in [12-14, 20]. The Lagrange method [20] is used to solve the system (6), (7). The area on the surface of the water occupied by the light liquid is represented as an ensemble of $N_{p}$ particles. For the numerical solution of the system (1-2), a second-order cross-over scheme is used.

\subsection{Informational system design for monitoring of oil spills}

Informational systems (IS) are created to monitor the state of oil spills on the basis of data on external environmental conditions [21]. In this work, IS is implemented as a Windows 
application for use on a personal computer and makes recommendations on the use of an artificial dispersant to remove oil spill residue. The control system for data bases (CSDB) for IS is chosen to be the SQLite associated with a relational model of data derived from model calculations. When analyzing the subject area, input and output data, an info logical model of the database in the form of an ER-diagram was developed. The ER-diagram is developed taking into account the semantics of the subject area. A user interface has been developed for operational monitoring of the oil spill. The developed interface is convenient, intuitive and is installed on a compact media such as a laptop. When you define selection parameters, you mainly use combined lists, which allows you to quickly select the required parameter or enter it from the keyboard. This reduces possible input errors. To develop the application, the Python programming language is selected. It has the following main advantages: free license, cross-platform, a large number of libraries.

The basic information for making a decision is the date, time and place of the spill, oil or oil product discharge regime, type of oil, rheological characteristics of oil, water salinity, water and air temperature, wind speed, height of waves. The result of the program is information about the possibility or impossibility of using dispersants at a particular combination of parameters, the preferred form of dispersant (liquid, dry or paste), the method of using and the amount of dispersant added. To calculate the amount of dispersant, a semi-empirical ratio [22] is used, defined as the ratio of the mass of the remaining oil on the surface to the value of the dispersant capacity. The dispersing capacity is calculated based on experiments to determine the dispersant efficiency depending on the temperature, salinity and dilution of the dispersant. The dispersant-oil ratio as a whole ranges from 1:3 to $1: 10$, decreasing with increasing ambient temperature.

The modular construction principle, which, in part, has been implemented, allows independent development of parts of the system, as well as, if necessary, replacing models with better and faster ones without significant architectural changes.

\section{Results}

The mathematical model of the formation of surface waves and its experimental verification were partially presented in our work [23]. The results of the surface wave model calculations show that this model satisfactorily describes the evolution of a single wave crest. Numerical integration of the equations of motion (4) is performed by a modified Euler-Cauchy method, which for one of the spatial directions can be written as follows [15]. A Fortran 95 program is written for the numerical integration.

The number of elements in the model $\mathrm{N}$ is from 5000 to 50000 , depending on problems to be solved. As an example, there are some results for data as follows: $N_{e}=5000$; $k=5 \cdot 10^{5} \mathrm{~kg} / \mathrm{m} \cdot \mathrm{sec} ; \mu=10^{-1} \mathrm{~kg} \cdot \mathrm{m} / \mathrm{sec}^{2} ; d_{e}=10^{-3} \mathrm{~m} ; d_{0}=1.1 \cdot 10^{-3} \mathrm{~m} ; d_{1}=10^{-3} \mathrm{~m}$.

The results of calculations for the model (4) are presented on Figures 1 - 4.

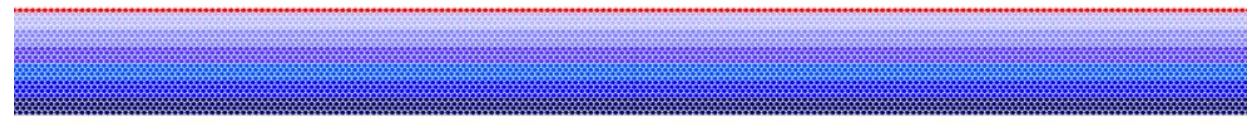

Fig. 1. Initial state without waves. 


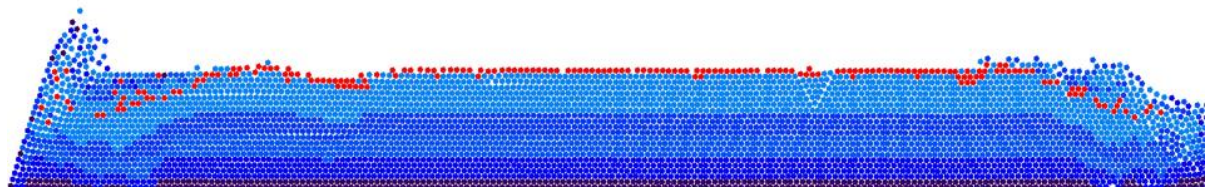

Fig. 2. Wave formation at $A=1.0 \mathrm{~m}$.

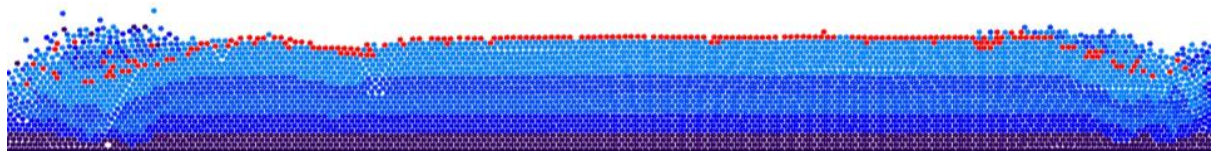

Fig. 3. Oil slick destruction at $A=1.0 \mathrm{~m}$. Red points represent particles of oil slicks.

Figure 1 shows an initial state, the oil slick particles are presented as black balls on the water surface, and stratification of water layers is represented by shades of gray.

Then, as a result of dispersion by wave motion, oil particles penetrate deep into the water, but the bottom layers of water remain undisturbed, as it can be seen on Figure 2-3. The black polyline in this drawing represents the boundary between floating oil particles on the surface and sunken ones. Next, Figure 4 shows a diagram of the distribution of oil remaining on the surface (white columns of the diagram) and dispersed oil (black columns). The levels are counted up and down from the black polyline in the previous drawing. The extreme columns of the chart include the number of particles below or above this level, respectively. The ratio of the number of sunken oil particles to their total number is 0.247 (24.7\%).

HISTOGRAM Of PARTICLES

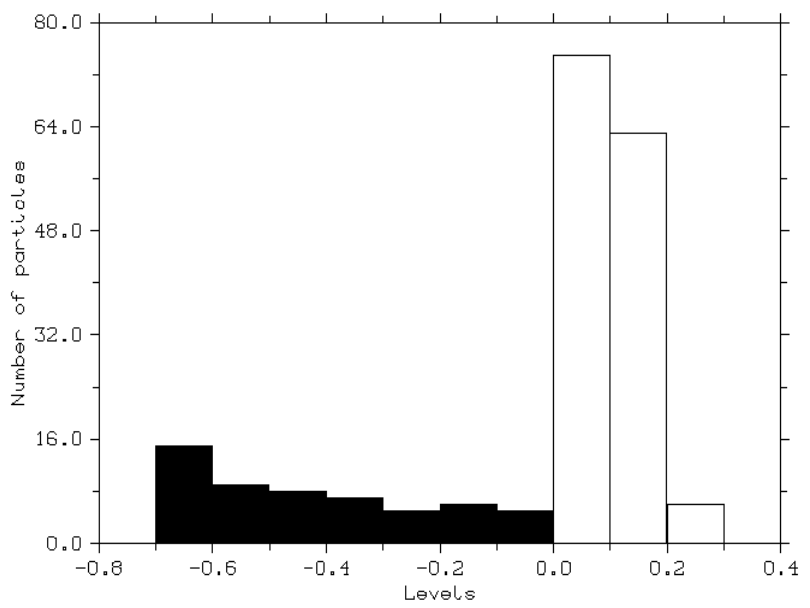

Fig. 4. Diagram of oil particles distribution by levels.

The mathematical model of the transfer and evolution of the oil slicks on the surface of the sea area is implemented in the form of a calculation program in the Fortran 95 programming language. The program implements a numerical model of the evolution of the 
spill of oil and oil products on the water surface. The program is a console application that executes in batch mode. The input information is information on the oil spill: characteristics of the discharged oil product, including its physicochemical properties, including fractional composition, as well as characteristics of the discharge source and information on the state of the environment.Some calculated results of the spill integral characteristics evolution are presented on Figure 5-6. The data, the spill mass balance and the slick area namely, are supposed to be recorded at intervals of one hour.

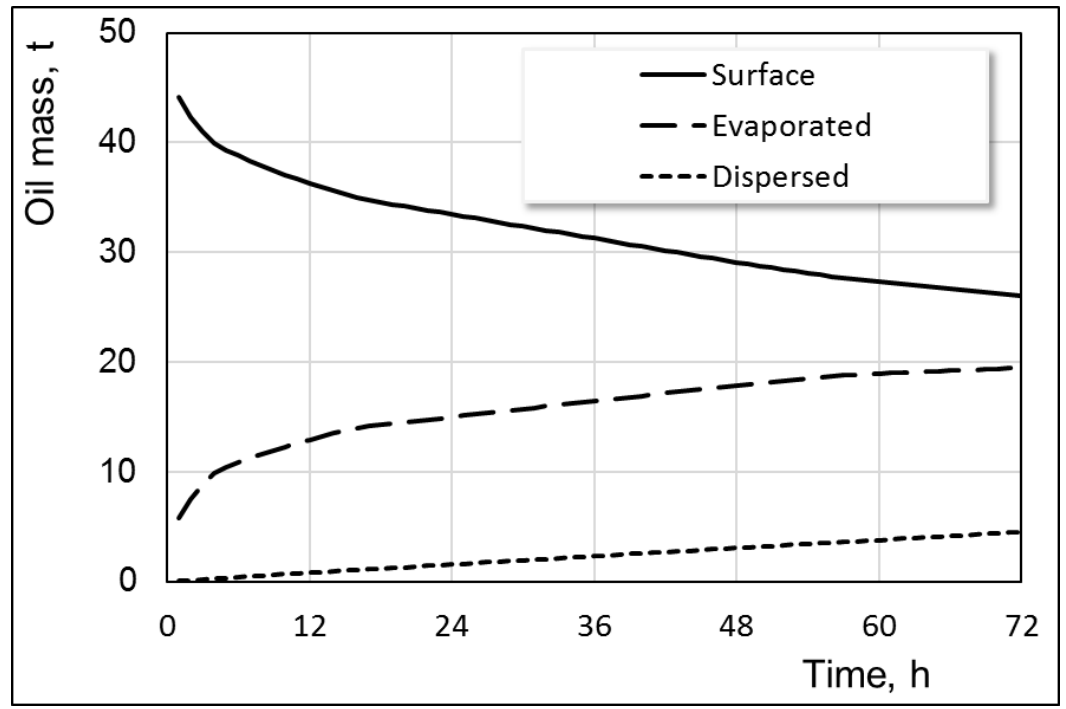

Fig. 5. Spill mass balance.

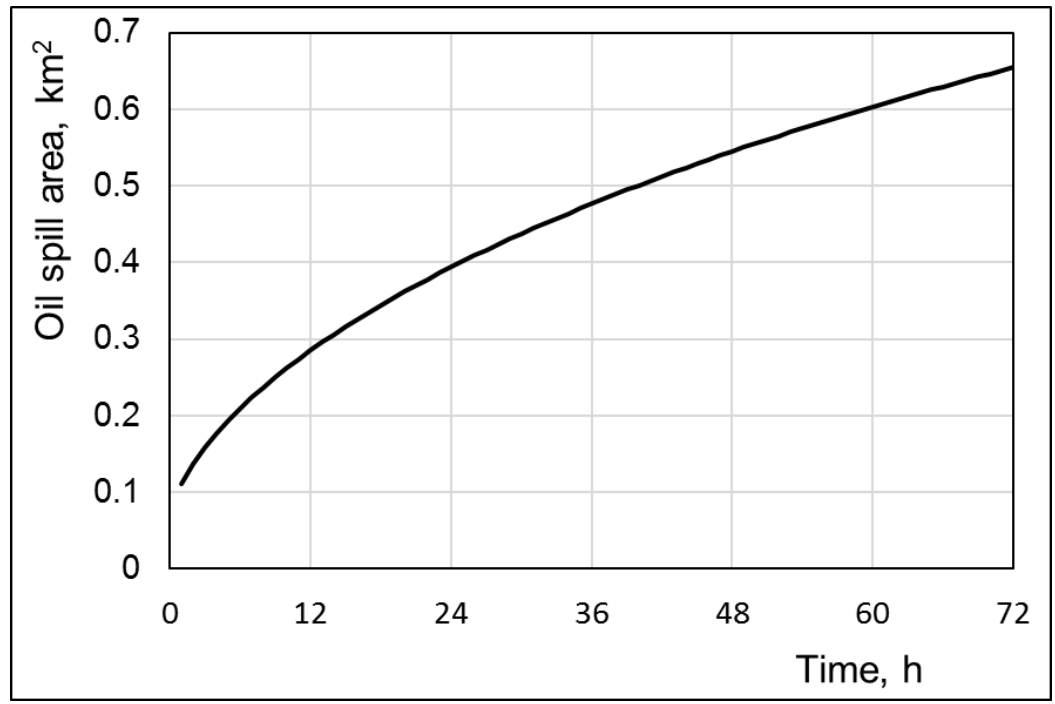

Fig. 6. Evolution of the spill area.

Figures 7 (a-b) show the thickness of an oil slick in the form of isolines at different times. As the analysis of the calculation results showed, the ambient temperature, wind speed and physical properties of oil products have most impact on the processes of destruction of an oil slick. 


\section{Distance, $m$}

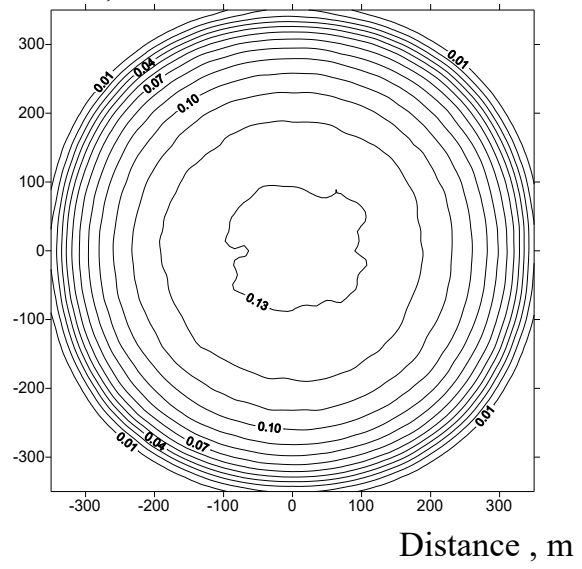

a)
Distance, $\mathrm{m}$

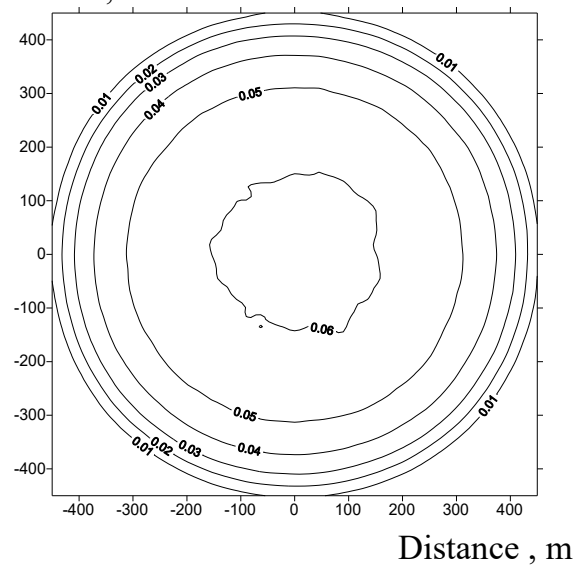

b)

Fig. 7. Oil thickness field (mm), a) - 24 hours after the start of the oil spill, b) - 72 hours after the start of the oil spill.

Water salinity is also an important factor. For instance (in the case of Urals type oil with wind of $5 \mathrm{~m} / \mathrm{s}$ by temperature $\mathrm{T}=5^{\circ} \mathrm{C}$ ) the amount of dispersed oil under fresh water conditions after 72 hours would be $4542.5 \mathrm{~kg}$ of the total amount of $50000 \mathrm{~kg}$ of spilled oil, and under conditions of sea water salinity of $3 \mathrm{ppm}$ the same would be $4440.1 \mathrm{~kg}$, i.e., decreased by $2.3 \%$.

\section{Discussions}

Modeling of oil and petroleum products spills, as well as the use of models of their behavior, in environmental conditions is one of the important factors in making decisions on liquidation of pollution and forecasting of the state of the environment exposed to pollution from oil spills $[21,24,25]$. One of the factors affecting the removal of petroleum products from the surface of the water area is natural dispersion due to surface mixing. [23]. Model of surface waves can be considered as a superstructure (mesoscale model) for simulating surface oscillatory motion. The need to consider such a model is that it provides quantitative estimates of oil dispersion due to surface wave effects on the oil spill spot. It is noted [10] that at a wind speed of more than $12 \mathrm{~m} / \mathrm{s}$ due to strong waves, the dispersion of "oil in water" occurs quickly enough and the emulsion of water in oil is almost not formed. Unlike the model [16], we assume that, firstly, the size of the elastic interaction region differs from the size of the dissipative interaction region, and secondly, we significantly change the mechanism of excitation of surface waves. In our model, we only perturb the upper layers of the liquid, which is more consistent with the idea of surface fluctuations in marine areas, where the perturbing force is provided by wind load. Calculation of oil evolution on sea surface is based on solution of two-dimensional equations of motion and continuity for oil slicks. The desired variables are the thickness of the oil layer and the speed of movement. The motion of the oil film is caused by the gradient of the oil thickness, the friction stress of wind and water at the respective boundaries. Oil is represented as multicomponent mixture, evaporation of which depends on characteristics of components and their content in mixture [24]. Unlike [17-19], time calculations use splitting by physical processes. At the first stage, a time step is made for the transfer and spreading process, at the second evaporation dispersion and emulsification are calculated. 


\section{Conclusions}

Artificial dispersants currently used to control oil spills are specially designed mixtures that include surfactants with solvents and other chemical components. Detailed analysis of efficiency and emerging problems when using chemical dispersants is given in the work [24]. The disadvantage of using chemical dispersants is their environmental hazard for marine organisms living in the depths of the water area where chemical dispersants are used. The work [25] provides an overview of methods of oil spill elimination based on the use of dispersants consisting of fine particulate matter. In the case of powdered dispersants based on natural compounds that do not contain synthetic highly toxic chemical compounds, environmental effects are expected to be reduced compared to other formulations. The most effective solution seems to be the use of artificial powder dispersants in the form of an emulsion, the calculation of which depends on the external conditions mentioned above. The ultimate goal in eliminating the spill site is to remove the oil spot from the surface of the water area. Since the most important factor in the oil spill degradation process is the dispersion of oil in the water column by both natural surface mixing and artificial dispersants, it is necessary in the calculation of the dispersant amount to take into account the determining external factors.

The reported study was funded of RFBR according to the research project No. 19-07-00493.

\section{References}

1. C.H. Barker and all., J. of Marine Sci. and Eng, 8, 9 (2020)

2. G. Simon, F. Dumas, T. Dohaut, J. Ocean Dynamics, 61 (2011)

3. K. Hasselmann, Geophys. Astrophys. Fluid Dynamics, 1, 3 (1970)

4. P.K. Kundu, J. Phys Oceanogr, 10, 2 (1980)

5. P. Lazur, F. Dumas, Adv. Water. Resour., 31, 2 (2008)

6. P. Lazur, V. Garnier, F. Dumas, C. Herry, M. Chifflet, Cont. Shelf Resour, 29, 8 (2009)

7. L.N. Ly, R. Garwood, J. Oceanography, 56 (2000)

8. S. Ermakov, I. Kapustin, O. Shomina, Sovremennye problemy distantsionnogo zondirovaniya Zemli iz Kosmosa, 12, 1 (2015)

9. M. Donelan, F. Dobson, S. Smith, R. Anderson, J. Phys. Oceanogr., 25 (1995)

10. W. Drennan, M. Donelan, E. Terray, K. Katsaros, J. Phys. Oceanogr, 26 (1996)

11. S. Kitaigorodskii, Tellus, 46A (1994)

12. G. Delvigne, C. Sweeney, Oil Chem. Pollut, 4, 4 (1988)

13. M. Reed and all., Spill Sci. Technol. Bull, 5, 1 (1999)

14. R. Miranda, WIT Trans. Ecol. Environ, 40 (2000)

15. H. Gould, J. Tobochnik, W. Christian, An Introduction to Computer Simulation Methods (New York, Wiley-Interscience, 2005)

16. A.V. Kalach, O.V. Shcherbakov, Vestnik Sankt-Peterburgskogo Universiteta Gocudarstvennoi Pozharnoi Sluzhby MChS Rossii, 4 (2013)

17. L.A. Brovchenko, V.S. Maderich, Priklanaya gidromekhanika, 6, 76 (2002)

18. W.C. Yang, H. Wang, Water Research, 11, 10 (1977)

19. P. Sebastiao, C. Soares, Spill Sci. Technol. Bull., 2, 2 (1995) 
20. S.N. Zatsepa, A.A. Ivchenko, V.V. Solbakov, Science and Methodological recommendations (Moscow, AS "Finpop", 2018)

21. E.V. Nikolaeva, M.A. Troshin, I.R. Aysmatullin, A.N. Radchenko, Science \& Technologies, Oil and Oil Products Pipeline Transportation, 8, 5 (2018)

22. S.V. Matsenko, G.G. Volkov, N.A. Volkova, Methodological recommendations (Novorossiysk, Moscow State University named after Adm. F.F. Ushakova, 2009)

23. E.V. Nikolaeva, S.L. Rychkov, A.V. Shatrov, IOP Conf. Ser., Earth Environ. Sci., 666 (2021)

24. G.N. Semanov, A.N. Gutnik, S.N. Zatsepa, A.A. Ivchenko, V.V. Solbakov, V.V. Stanovoy, A.S. Shivaev, Arctika, Ecologia and Economika, 2, 1 (2017)

25. A.V. Sal'nikov, M.A. Troshin, E.V. Nikolaeva, Science \& Technologies, Oil and Oil Products Pipeline Transportation, 9, 6 (2019) 